\title{
FORMACIÓN Y VALORACIÓN DE PROFESORES EN EL PLAN NACIONAL DE EDUCACIÓN (BRASIL/ 2014-2024) Y EN EL PLAN DE EDUCACIÓN DE ANDALUCÍA/ ESPAÑA (2014): APROXIMACIONES Y DISTANCIAMIENTOS
}

Iria Brzezinski

Carlos Marcelo García

RESUMO: Configuram-se como objeto da pesquisa que oferece sustentação a este artigo as aproximações e distanciamentos em dois planos de educação: o do Brasil sancionado por meio da Lei n 13.005, de 25 de junho de 2014 e o III Plan Andaluz de Formación del Profesorado (Espanha), aprovado pela Orden de 31 de julio de 2014 no tocante à formação e valorização de professores. A investigação desenvolveu-se sob uma perspectiva socio-histórica, mediante uma abordagem qualitativa de caráter teórico, com análise documental e estudos comparados.

PALAVRAS CHAVE: Políticas de formação e valorização dos Professores; Plano Nacional de Educação: 2014-2024, Plano Andaluz de Formação do Professorado.

RESUMEN: El objeto de la investigación presentada en este artigo son las aproximaciones y los distanciamientos en dos planes de educación: el de Brasil, sancionado por la Ley n. 13.005, de 25 de junio de 2014, y el III Plan Andaluz de Formación del Profesorado (España), aprobado por la Orden de 31 de julio de 2014, en lo que se refiere a la formación y valoración de profesores. La investigación se desarrolló bajo una perspectiva socio-histórica, mediante un abordaje cualitativo de carácter teórico, con análisis documental y estudios comparados.

PALABRAS CLAVE: Política de Formación y Valoración de los Profesores; Plan Nacional de Educación: 2014-2024; Plan Andaluz de Formación del Profesorado

\begin{abstract}
Configured as an object of research that offers support to this article the similarities and differences in two education plans: Brazil's plan sanctioned by Law No. 13.005 of June 25, 2014 and the III Plan Andaluz de Teacher's formation (Spain), approved by the Order of July 31 of 2014 in relation to the training and development of teachers. The research was developed in a socio-historical perspective through a qualitative approach of theoretical character, with document analysis and comparative studies.
\end{abstract}

KEYWORDS: Training and Improvement of Teacher Policy; National Education Plan: 2014-2024; Plan Andaluz of Teacher's formation. 


\section{autêntica}

\section{CONSIDERACIONES INTRODUCTORIAS}

Formamos un par de profesores que se dedican a los estudios e investigaciones acerca de Políticas de Formación de Profesores y valoración del trabajo docente.

En este artículo, osamos enfrentar el desafío de desarrollar estudios comparados entre formación y valoración de profesores según lo que consta en el Plan Nacional de Educación (2014-2024/BRASIL), Ley n 13.005, de 25/7/2014 y en el III Plan Andaluz de Formación del Profesorado, Orden de 31/7/2014 y Ley 17, de 10/12/2007 (ESPAÑA. Andalucía).

Desde el inicio, establecimos diálogos con nuestros interlocutores. Ideas, argumentos y análisis comparativo son la base epistemológica de mis trabajos científicos que se asientan, en especial, en mi formación inicial como científica social y pedagoga. La formación continuada a lo largo de la vida profesional discurre en dos caminos que se entrecruzan: uno, recorrido como profesora e investigadora dedicada a los estudios en el campo de las políticas educativas, de formación y valoración de profesores, y de la evaluación institucional; y otro, recorrido como militante en defesa de la escuela pública, laica, gratuita en todos los niveles y modalidades de enseñanza para todos los brasileños. Milito en entidades científicas que comulgan objetivos en favor de la calidad social en la formación de profesores y de la valoración de los profesionales de la educación.

En este espacio-tiempo, me considero sintonizada con el ideario y me incorporo a las luchas y manifiestos, proyectando utopías y realizaciones del Fórum en Defensa de la Escuela Pública y de las asociaciones. Soy dirigente en los colegiados de la ANFOPE - Asociación Nacional por la Formación de los Profesionales de la Educación y de la ANPEd - Asociación Nacional de Postgrado e Investigación en Educación.

Ese campo de lucha, de muchas indignaciones, características de los movimientos sociales organizados que se manifiestan como resistencia al poder constituido, es marcado por intensos períodos de avances - conquistas - y retrocesos - frustraciones. Como declara Castells (1999, p. 419-420), esas asociaciones son "[...] comunidades formadas a partir de la identidad de resistencia". Se trata de resistencia activa de la sociedad civil expresada en una verdadera arena política, en que las fuerzas sociales se organizan y contribuyen para el trazado, la ejecución y evaluación de políticas educativas.

Como profesor, investigador y formador, me ha preocupado mejorar las condiciones y la práctica del aprendizaje de los profesores. Participo en los procesos de creación y formación de la red de asesores de formación en los centros del profesorado y, desde el comienzo de nuestro trabajo, nos identificamos con una visión del trabajo en la universidad, dialogando con las personas que desempeñan la importante labor docente. Mi interés ha sido combinar de forma equilibrada la vida académica universitaria y el trabajo con los docentes. He aportado ideas que ahora son una realidad pero que cuando las propusimos resultaron extrañas tanto en España como en América Latina. En especial, la preocupación por la formación y el acompañamiento del profesorado principiante. Nuestro primer trabajo en este terreno data de 1991 y desde entonces hasta ahora hemos conseguido poner en la agenda política esta necesidad gracias entre otras cosas a los Congresos que organizamos cada dos años sobre el profesorado principiante.

La universalidad de nuestra investigación recae en documentos de políticas de la educación con foco en la Planificación Educacional del Brasil y de Andalucía/España.

Las especificidades consisten en el Plan Nacional de Educación del Brasil (PNE 2014-2024), en particular, en las estrategias y metas que tienen por objeto la formación y la valoración de los profesionales de la 


\section{autêntica}

educación y en el Plan Andaluz de Formación del Profesorado. España es un país con bastante autonomía en sus territorios o comunidades autónomas. Andalucía ha generado una consistente política de valoración y formación de los docentes que se concreta en el III Plan Andaluz de Formación Permanente del Profesorado, que analizaremos en este artículo.

Nuestro itinerario investigativo se dirige a la comparación de las políticas de formación y valoración de profesores en el ámbito de la planificación educacional en los dos contextos anteriormente citados. Nuestra selección pretende lo siguiente: desarrollar estudios comparados, procurando revelar aproximaciones y distanciamientos. Nuestra base de investigación está en los ordenamientos jurídicos referentes a las políticas educativas pertinentes al campo de la planificación de la educación.

\section{ITINERARIO INVESTIGATIVO: FUNDAMENTACIÓN TEÓRICA Y METODOLOGÍA}

Esta investigación es cualitativa, teórica, con análisis documental y estudios comparados. Encontramos en ZEMELMAN (2003) subsidios que justifican los estudios, pues tales investigaciones consideran las especificidades históricas en la medida en que contextualizan y marcan realidades culturas diferentes.

Ese procedimiento de investigación requiere referencias del contexto, de la delimitación e interpretación de las normas de los planes en análisis, para a posteriori, realizar la comparación que deberá posibilitar una visión comprensiva epistemológica, conceptual y metodológica en la medida en que permite desvelar un determinado contexto en relación al otro (MENEZES, JUNIOR; BRZEZINSKI, 2015).

Garrido García (1991, p.157) estudioso de Trojan y de Sanchez (2009) aclara que “para la educación comparada, aunque se contemplen diferentes enfoques, su objeto comprehende los sistemas de educación, o la consideración del proceso de educación en sistemas sociales concretos".

El mismo autor traza una reconstitución histórica de los estudios comparados y toma como referencia a Sadler (1964) que reconoce la configuración del Estado-Nación, al inicio del Siglo XX, como un aporte para el desarrollo de esas investigaciones:

El verdadero valor de los estudios comparados no reside en descubrir dispositivos o mecanismos que puedan ser transferidos de un país para otro (aunque no deba ignorarse la frecuente posibilidad de tal transferencia), sino en comprobar cual es el espíritu que ha engrandecido la institución extranjera, para luego buscar medios de cultivar ese espíritu en el seno del propio país, en el caso de que se juzgue necesario para reparar algún fallo en la vida nacional (SADLER, 1900, p. 56 apud GARCÍA GARRIDO, 1996, p. 54).

En la contemporaneidad, los estudios comparados se insertan en el contexto del sistema social y económico mundial, que lanni (1996, p. 97) denomina "aldea global". En ella "todo se globaliza y se hace virtual" [...] cada individuo puede ser un haz de articulaciones locales, nacionales, regionales y mundiales, cuyos movimientos y centros de emisión están dispersos y desterritorializados por el mundo". El autor resalta: "el principal tejido de la aldea global ha sido el mercado, la mercantilización, en el sentido de que todo tiende a ser mercantilizado, producido y consumido como mercadoría" (IANNI, 1996, p. 99).

Con la misma lógica interpretativa, Canário (2009, p. 356) señala que las políticas educativas bajo la tutela del neoliberalismo "se inscriben en la ideología hegemónica internacional, subordinándose [...] a los imperativos de carácter económico inherentes a un mercado global y único". 


\section{autêntica}

En esta coyuntura, Nóvoa (2009, p.24) destaca que la hegemonía internacional sobre los países periféricos obstaculiza el desarrollo "de la educación comparada entre los países de Occidente, una vez que legitima las acciones o que impone silencios, que se imita o que se coloniza".

Los estudios comparados, aquí construidos, tienen el vector diferencial entre Brasil y España: aquel es un país de modelo económico capitalista y régimen político republicano federal democrático representativo, mientras que esta es una monarquía constitucional y representativa. España es un país miembro de la Unión Europea, miembro del grupo de países que pertenecen al euro como moneda única. La zona de países que pertenecen al euro constituye una economía de mercado, capitalista, pero con atención a lo que se ha dado en llamar "estado de bienestar" donde el estado asume responsabilidades en aspectos relacionados con la educación, salud, dependencia de las personas y otras necesidades sociales como ayudas a desempleados, jubilados, entre otros.

Compartimos, aun, ideas de Wiseman (2010), de que a pesar de la singularidad de cada país, de políticas y sistemas nacionales, el impacto de homogenización de la globalización en la actualidad incide tanto como ideología como en la acción de reorganizar el Estado de Derecho, el poder y la soberanía de los gobiernos nacionales.

En el caso de España, su pertinencia a la Unión Europea y a la moneda única, así como a un espacio común de seguridad y justicia común, establece unos límites supranacionales al concepto de estado-nación. Los tratados internacionales que sirven de constitución a la Unión Europea (Tratado de Maastricht) establecen unos principios que es importante destacar (igualdad y ciudadanía europea, democracia representativa, transparencia y participación), que se concretan en un parlamento europeo, una Comisión Europea con funciones de establecimiento de políticas comunes y un Banco Central Europeo. Todas estas instituciones han ido progresivamente asumiendo funciones que anteriormente descansaban en los estados miembros.

Cuanto a la metodología y a los procedimientos de investigación, tomamos como referencia a Cowen (2012) que hizo un balance crítico sobre una buena parte de obras de investigadores de la educación comparada mundial. El autor señala que el desarrollo de estudios de educación comparada como disciplina o como metodología científica se revela marcado por continuidades y discontinuidades desde su origen, en el siglo XVII. Sugiere a los investigadores que ante tal contradicción las investigaciones con ese objeto sean orientadas por ideas-unidad: espacio; tiempo; sistema educacional; identidad educada; contexto social; transferencia; praxis (COWEN, 2012). Inspirados en esas ideas-unidad, optamos por tres de ellas, aunque adaptadas al marco de este trabajo: espacio-tiempo, contexto socio-político-económico y sistemas educativos. Este último fue escogida en particular debido a la implantación del PNE/Brasil que depende del régimen de colaboración entre entes federados, pues consisten en mecanismos de articulación del Sistema Nacional de Educación. Al mismo tiempo, esa idea-unidades es aplicada al contexto regional de la planificación para Andalucía.

El documento que vamos a describir en relación con Andalucía es el III Plan Andaluz de Formación Permanente del Profesorado. El hecho de ser el tercero significa la obviedad de que ha habido dos planes anteriores. Esto supone que Andalucía ha venido diseñando políticas públicas para consolidar una estructura de formación continua de los docentes.

La Ley de Educación de Andalucía (2007) establece en su art. 19 lo siguiente:

1. La formación permanente constituye un derecho y una obligación del profesorado. A tales efectos,

la Consejería competente en materia de educación realizará una oferta de actividades formativas diversificada, adecuada a las líneas estratégicas del sistema educativo, a las necesidades demandadas 


\section{autêntica}

por los centros en este ámbito y al diagnóstico de necesidades que se desprendan de los planes de evaluación desarrollados.

2. Las actividades de formación permanente del profesorado tendrán como objetivo el perfeccionamiento de la práctica educativa, de forma que incida en la mejora de los rendimientos del alumnado y en su desarrollo personal y social, a través de la atención a sus peculiaridades y a la diversidad del mismo.

3. Las modalidades de formación del profesorado perseguirán el aprendizaje de las buenas prácticas docentes, el intercambio profesional y la difusión del conocimiento que contribuya a la creación de redes profesionales. Las estrategias formativas estimularán el trabajo cooperativo a través, fundamentalmente, de la formación en centros y de la autoformación, y tendrán en cuenta los distintos niveles de desarrollo profesional del profesorado.

Como consecuencia de lo anterior, hay que entender que la formación permanente del profesorado en Andalucía, al ser un derecho del profesorado, es completamente gratuita. La formación permanente del profesorado en Andalucía se estructura en torno a los Centros del Profesorado. Estos se crean en Andalucía en 1986. Son instituciones que, aunque dependen de la administración educativa autonómica, poseen un funcionamiento y organización bastante autónomos. Estos centros tienen una estructura descentralizada, ya que en cada provincia hay varios, dependiendo de la extensión de la misma. Cuentan con un plantel de formadores, denominados asesores, que desempeñan tareas formativas en diferentes modelos de formación. Los asesores son docentes que han participado en proyectos de innovación educativa y que dejan provisionalmente la docencia para implicarse en las tareas formativas. Hay que decir que en esta formación las universidades formalmente no participan, sino que está gestionada por el propio profesorado.

Una de las características de la formación permanente en Andalucía es la variedad de sus modalidades de formación. Así, junto a los cursos de formación ha ido creciendo una oferta formativa relacionada con proyectos de innovación, grupos de trabajo, proyectos de formación centrada en la escuela, proyectos de mejora escolar. En este tipo de modalidad de desarrollo profesional, son los propios centros educativos y sus profesores los que deciden las temáticas de formación o innovación, los que se organizan y participan en los proyectos, con el apoyo de los asesores de los Centros del Profesorado. El III Plan Andaluz establece prioridades políticas para la mejora de las estructuras y funcionamiento de la formación que se realiza en los Centros del Profesorado.

Para justificar nuestra elección de los estudios comparados contribuyeron las investigaciones de YANG (2015, p. 319), ya que el autor profundiza en los "aspectos teóricos y metodológicos del análisis comparado de políticas educativas." En lo que se refiere a la política educacional, aseguramos que ella no resulta de la exclusiva deliberación de los que ejercen el poder en la máquina burocrática del Estado democrático, como Brasil. La política educacional es fruto de la compleja relación que se establece entre el Estado y las fuerzas sociales en un Estado Mínimo regulado por el mercado, imputando a los trabajadores muchas pérdidas y raras conquistas.

Cuanto a la elaboración de una política, Yang (2015, p. 322) se remite al trabajo de investigadores que

puede englobar un ámbito muy amplio y ser comprendido y usado de varias maneras [por ejemplo] en planes, decisiones, documentos y propuestas. [...]. La definición más popular entre los investigadores de políticas y el público en general es la que define políticas como si fueran documentos. 


\section{autêntica}

Yang señala que los documentos de políticas pueden asumir diversas formas y niveles diferentes. En nuestro caso, procedemos a estudios de dos planes de formas y niveles distintos: un brasileño de ámbito nacional, el otro español, relativo a Andalucía. A seguir pasaremos a su análisis.

\section{ITINERARIO INVESTIGATIVO: PNE (2014-2024), POLÍTICAS EDUCACIONALES BRASILEÑAS, DERECHO A LA EDUCACIÓN Y A LA CIUDADANÍA EN EL ESTADO DEMOCRÁTICO REPUBLICANO}

Los documentos-base de política educacional brasileña aquí analizados son la Constitución Federal (CF) de 5/10/1988, la Ley de Directrices y Bases de la Educación Nacional (Ley n. 9.394, de 20/12/1996) y, específicamente, el PNE (2014-2014). De la CF/1988 nos interesan los artículos 1º, 208 y 214 y, de la LDB/1996, el art. $4^{\circ}$ y art. $5^{\circ}$.

Destacamos que los Constituyentes de 1988 divulgaron la concepción y la finalidad de la educación en el art. 205, entendiendo la educación como "derecho de todos y deber del Estado y de la familia", a ser "promovida con la colaboración de la sociedad"; con las precipuas finalidades de "pleno desarrollo de la persona, su preparación para el ejercicio de la ciudadanía y su cualificación para el trabajo".

La propia concepción y las finalidades de la educación en la Carta Magna suscitan la obligatoriedad de la inclusión de todo ciudadano brasileño en la educación básica pública - legalmente integrada desde los 4 a los 17 años de edad, siendo laica, gratuita e inclusiva.

La educación, constitucionalmente, consiste en principio y en derecho social asegurados en el art. 208 de la CF/1988 y en los art. $2^{\circ}$, art. $3^{\circ}$, art. $4^{\circ}$ y art. $5^{\circ}$ de la LDB/1996. El ciudadano independientemente de clase social tiene derecho al ingreso con igualdad de condiciones para el acceso y la permanencia con éxito en la educación básica.

Ese derecho social es de obligatoriedad del Estado, con la oferta de la educación básica integrada con 13 años de estudios. Esa fue una conquista mediante acciones combativas de los sujetos sociales colectivos (sociedad civil organizada en asociaciones, entidades y sindicatos) y de la lucha de clases en el espacio de la sociedad capitalista.

Ante la obligatoriedad de que todo ciudadano frecuente con éxito la educación básica, como determinación constitucional, los derechos deben ser garantizados tanto por fuerza de ley, como por la aplicabilidad de esa legislación, considerando que el Estado brasileño mantiene una organización política que se expresa en una República Federativa Democrática y Representativa.

En lo que se refiere a la democracia nuestro referencial se base en Chaui $(2008)$, Coutinho $(2008,1997)$ y Dias (1997). En conformidad con nuestro análisis, sus teorizaciones se articulan y se complementan. La primera autora entiende que

la democracia es la sociedad verdaderamente histórica, esto es, abierta al tiempo, a lo posible, a las transformaciones y a lo nuevo. Efectivamente, por la creación de nuevos derechos y por la existencia de los contrapoderes sociales, la sociedad democrática no está fijada en una forma para siempre determinada, o sea, no cesa de trabajar sus divisiones y diferencias internas, de orientarse por la posibilidad objetiva (la libertad) y de alterarse por la propia praxis (CHAUI, 2008, p. 406) (grifos de la autora).

Chaui nos aclara que los cambios provocados en la praxis de la democracia en un tiempo y espacio históricos determinados son posibilidades política y social objetivas para que el sujeto, en la comunidad, conquiste 


\section{autêntica}

el ejercicio pleno de la ciudadanía con la práctica de la libertad individual y colectiva, en consecuencia del respeto a los derechos humanos, que abarcan los derechos civiles, económicos, sociales y culturales.

De acuerdo con Coutinho (2008, p. 50), en el Estado democrático representativo "democracia es sinónimo de soberanía popular". La democracia se manifiesta por el respeto "a las condiciones sociales e institucionales que posibilitan al conjunto de ciudadanos la participación activa en la formación del gobierno y, como consecuencia, en el control de la vida social".

En lo que se refiere a la ciudadanía, el autor insiste en la defensa de que en un Estado democrático su presencia se hará efectiva cuando todos los ciudadanos se "apropien de los bienes socialmente criados, de actualizar todas las potencialidades de la realización humana abiertas por la vida social en cada contexto históricamente determinado" (ibíd.).

Efectivamente, "solo puede haber democracia para las grandes masas de la población si ellas fueran capaces de organizarse, de expresar sus deseos y de obtener efectivamente conquistas sociales, culturales y políticas en el cuadro de una institucionalidad en permanente expansión (COUTINHO, 2008, p.131).

Destacamos, sin embargo, que esa lucha es tensionada por avances y retrocesos, en razón de que el Estado mínimo mantiene las desigualdades históricas vividas por la clase que "vive-del-trabajo". No hay dudas de que la fase actual del "capital-imperialismo" [con] "predominio del capital monetario"; "dominación de la propiedad capitalista" [y el] "impulso [...] expropiador" (FONTES, p. 149) de los saberes de la clase popular acaban reduciendo el amplio espectro de la democracia al componer "un estándar bifurcado de actuación política, altamente internacionalizado para el capital y fuertemente fragmentado para el trabajo" (ídem).

Todavía acerca de los derechos a la ciudadanía, aunque haya el reconocimiento por la clase dominante de las conquistas. Coutinho (1997, p. 157) asevera que "eso no anula la posibilidad de que, en determinadas coyunturas, a depender de la correlación de fuerzas, la burguesía use las políticas sociales para desmovilizar la clase trabajadora, para intentar cooptarla [....]". Ejemplo típico de tal cooptación, concretamente, de los sindicatos y confederaciones de la categoría de los trabajadores, ocurrió en el gobierno de Luiz Inácio Lula da Silva (período 2002-2008).

Bajo esta misma lógica de Chaui y Coutinho, la tematización sobre la falta de respeto a la ciudadanía en Brasil es enfatizada por Dias (1997, p.28) en razón de que la democracia "se encuentra reducida a una igualdad formal, en la que todos son considerados iguales ante la ley". La clase dominante, no obstante, protagonizada por el "individuo-ciudadano" y empresario-ciudadano usurpa de la clase dominada su condición de existencia. En ese proceso, con la finalidad de preservar sus riquezas materiales y simbólicas, la clase privilegiada abstrajo la identidad de la otra clase, sus instrumentos de producción y sus saberes.

El gobierno democrático y popular brasileño, en el período 2012-2014, promovió alguna movilidad social de la clase popular alzándola al status D y C, aunque bajo la tutela del régimen capitalista y del Estado mínimo. Entretanto, mantuvo casi intocadas las disparidades regionales que desvelan la existencia de muchos brasiles, en la geopolítica brasileña, en la cual la división social de clases es asimétrica, marcada por la injusticia social que impide la superación del analfabetismo, del desempleo, del hambre, de la miseria.

Hacemos justicia al recordar que, a partir del final de 2014, las clases sociales sufrieron con la recesión económica y la crisis política imputó pérdidas a toda la población. Ello resultó para el país, en 2015, en la pérdida de status de $7^{a}$ economía mundial (EXAME, 2015), impactando negativamente en el grado de riesgo Brasil 


\section{autêntica}

“[...] que actúa como un termómetro indicador de la confianza del mercado con relación a la economía del país (BRASIL, 2016).

El diálogo con los ciudadanos de la sociedad civil, como nuevos protagonistas del momento histórico contemporáneo brasileño debe ser necesariamente (re)establecido por el Estado republicano democrático. La relevancia de la participación social de esos sujetos en la esfera pública, por ejemplo, vía fórums, consejos, comités de gobernanza, "se hace para democratizar la gestión de la cosa pública, para invertir las prioridades de las administraciones en el sentido de políticas que atiendan no apenas las cuestiones emergentes" (GOHN, 2004, p. 20).

Esperamos que el PNE (2014-2024), ejecutado a largo plazo, dimensionado en metas y estrategias, venga a superar las prácticas de programas puntuales, considerados emergentes de formación y de valoración de profesores. Trataremos específicamente del PNE (2014-2024), que nos interesa como instrumento definidor de política educacional, a ser analizada en el ítem a seguir.

\subsection{PNE 2014-2015: FORMACIÓN Y VALORACIÓN DE PROFESORES}

Tal como fue señalado antes, la movilización de las fuerzas sociales en lo que concierne a la elaboración de las metas sobre formación y valoración de profesores fue muy intensa. La Anfope presentó un total de 96 enmiendas a la Comisión de Educación de la Cámara por intermediación de los diputados federales, entre ellas, 44 sobre formación y valoración de profesores(as). Registramos que las enmiendas fueron elaboradas en colaboración con otras entidades de estudios e investigación en educación.

Para la meta 15 fue elaborada una enmienda sustitutiva. Transcribimos la redacción dada por el relator y la enmienda de la Anfope.

Meta 15: garantizar, en régimen de colaboración entre la Unión, los Estados, el Distrito Federal y los Municipios, en el plazo de 1 (un) año de vigencia de este PNE, la política nacional de formación de los profesionales de la educación de que tratan los incisos I, II y III del inicio del art. 61 de la Ley n 9.394, de 20 de diciembre de 1996, asegurado que todos los profesores y las profesoras de la educación básica posean formación específica de nivel superior, obtenida en curso de licenciatura en el área de conocimiento en que actúan (BRASIL, 2014).

Enmienda Sustitutiva a la Meta 15: Implantar el Subsistema Nacional de Formación y Valoración de los de Profesionales de la Educación (SNFVPE), congregando un grande esfuerzo nacional para la formación docente, presencial, en actuación conjunta de los entes federados, estableciendo un plan estratégico que presente diagnóstico de las necesidades de formación y de valoración de los profesionales de la educación y de la capacidad de atendimiento por parte de instituciones públicas de educación superior existentes en los Estados, Municipios y Distrito Federal, y defina obligaciones recíprocas entre los partícipes, considerando la obligatoriedad de la implementación de la Ley n. 11.738/2008 que instituye el salario mínimo nacional para los profesionales del magisterio (BRZEZINSKI, 2011, p. 2).

Tal enmienda no fue acatada por el relator, así como las estrategias pertinentes a la meta 15. Caso fuera aceptada sería obligatoria la elaboración de una política global de formación inicial y continuada de formación de profesores, articulada a los planes de cargos y salarios y al atendimiento de la Ley del Salario Mínimo Nacional, base inicial para dignificar social y económicamente la profesión-profesor en el Brasil. 


\title{
autêntica
}

La enmienda sustitutiva de la Anfope a la Estrategia 15.1 no fue acatada por el relator, no obstante fue resguardada por la CONAE/2010 en los siguientes términos:

\begin{abstract}
Estrategia 15.1) institucionalizar, en el plazo de un año de vigencia del PNE - 2011/2020, el Subsistema Nacional de Formación y de Valoración de los Profesionales de la Educación, con la formulación de una política nacional elaborada con planes específicos, que aseguren formación inicial presencial, admitiéndose educación a distancia solamente en locales de difícil acceso, y formación continuada con licencia remunerada en período lectivo. Asegurar también la construcción de un Referencial Curricular Nacional, en fórums constituidos para tal fin, inmediatamente tras la aprobación del PNE, con participación paritaria del número de representantes de la sociedad civil organizada en su composición, estableciéndose una periodicidad para que ellos ocurran regularmente, con financiamiento definido (BRZEZINSKI, 2011, p. 2).
\end{abstract}

La CONAE/2010 señaló de forma objetiva la implementación de procesos e instrumentos de elaboración del Referencial Curricular Nacional y, además, respetando la concepción de la Base Común Nacional (BCN) construida por la Anfope y expresada en los principios para la formación de todos los que aspiran a ser profesores: la) sólida formación teórica e interdisciplinar; b) unidad teoría-práctica; c) trabajo colectivo e interdisciplinar; d) compromiso social del profesional de la educación; y) gestión democrática; f) incorporación de la concepción de formación continuada; g) evaluación permanente de los cursos de formación de los profesionales de la educación.

La referencia a la BCN consta del Documento Final de la CONAE (BRASIL, 2010, p. 82):

Base común nacional balizadora de los contenidos esenciales de la formación del profesor y como parámetro para la definición de la cualidad, bien como ser resultado de la articulación necesaria entre el MEC, las instituciones formadoras y los sistemas de enseñanza.

Aseguramos que ese conjunto de indicadores de una política de formación y valoración puesto en práctica daría organicidad al SNFVPE que, por su vez, se articularía al Sistema Nacional de Educación.

El trazado de una política de formación de profesores(as), de iniciativa de la Secretaría de Articulación de los Sistemas de Enseñanza (Sase) está en discusión en las diferentes secretarías del MEC. El Fórum Nacional de Educación también se debruzó sobre análisis del SNE y resultó en una minuta propositiva, en debate desde 15/12/2015, orientada a la reglamentación de ese sistema, en 2016.

Mientras no sea establecido un SNFVPE en Brasil, con un planteamiento que contemple las necesidades de la educación básica brasileña incurriremos nuevamente en un equívoco, como son las políticas gubernamentales de las dos décadas del siglo XXI: la Unión, uno de los entes federados del SNE integrado por los Estados, Distrito Federal y Municipios que, en régimen de colaboración, desarrolla prácticas fomentadoras de políticas puntuales, con carácter de emergencia por medio de planes y programas dispersos, marcados por la falta de organicidad y desarticulación en el interior del propio MEC. Añadamos un agravante, pues no hay control social sistemático de esas "emergencias", lo que implica, en muchos casos, la malversación de recursos públicos.

Tal descaso de las autoridades ha llevado el país a arcar con una política de formación enredada por la ideología de los financiadores bajo un "modelo" de formación de profesores que consiste mucho más en conceder una certificación de lo que conferir una buena calificación referenciada en lo social a los actuantes en el sistema educacional y a los jóvenes, futuros profesores a ingresar en el sistema educacional público. 


\section{autêntica}

La Anfope se interpuso a las prácticas de formación y valoración de profesores evaluadas como inocuas, por razones repetidamente explicitadas con respaldo en su legitimidad de haber construido como entidad científica, a lo largo de 30 años, un pensamiento nacional brasileño en defensa de esos derechos del profesor.

Sobre la Estrategia 15.3, se reviste de gran significado por raramente haberse verificado en el país. Son políticas permanentes de iniciación a la docencia de estudiantes matriculados en cursos de formación de profesores, con el propósito de perfeccionar su práctica pedagógica para actuar en la educación básica. Son buenos indicios de política con calidad pedagógica y técnica, aunque no basta con fomentar programas de perfeccionamiento. Se hace imprescindible una política continua edificada por el SNFVPE.

Integrarían ese Sistema aun lo establecido en las metas 16 y 17, citadas a seguir.

Meta 16: formar, en nivel de postgrado, 50\% (cincuenta por ciento) de los profesores de la educación básica, hasta el último año de vigencia de este PNE, y garantizar a todos(as) los(as) profesionales de la educación básica formación continuada en su área de actuación, considerando las necesidades, demandas y contextualizaciones de los sistemas de enseñanza (BRZEZINSKI, 2011, p. 4).

Pairan dudas acerca de la viabilidad de esta meta. A pesar de su materialización prevista con plazo alargado hasta 2024, están implicados los siguientes condicionantes, entre otros:

a) cortes continuos de recursos vinculados a la educación;

b) grande contingente de personas que actúan como profesores en la Educación Básica, no obstante, son legos;

c) la herencia histórica a ser superada, aun garantizada por la Ley $n^{\circ} 12.796$, de 4/4/2013, que alteró la LDB/1996, o sea, la permanencia de la formación de profesores en nivel medio en la modalidad Normal (Magisterio) con derecho la ejercer la docencia en la educación infantil y en los años iniciales de la enseñanza fundamental $\left(1^{\circ}\right.$ al $5^{\circ}$ años).

Cuanto a la

Meta 17: valorar los(as) profesionales del magisterio de las redes públicas de educación básica de forma a equiparar su rendimiento medio al de los(as) demás profesionales con escolaridad equivalente, hasta el final del sexto año de vigencia de este PNE (BRZEZINSKI, 2011, p. 4).

Evaluamos como positivo el mérito de la valoración del magisterio al equiparar el salario del profesor al rendimiento medio de los "demás profesionales con escolaridad equivalente". Se configuró como derrota la lucha emprendida por las entidades y sindicatos de la categoría para que esa equiparación fuera materializada durante el primer año de vigencia de la Ley n. ${ }^{\circ}$ 13.005/2014. Lamentablemente, fue postergada y, caso ocurra, será solamente en 2020.

En lo que se refiere a otros aspectos de la valoración de los profesionales de la educación resaltamos el inicio de la meta 18, cuando asegura la existencia (en el plazo de dos años) de los Planes de Carrera para profesionales de la educación básica, lo que incluye la conquista de planes de carrera de los funcionarios de la educación básica. 


\section{autêntica}

Meta 18: asegurar, en el plazo de 2 (dos) años, la existencia de planes de Carrera para los(as) profesionales de la educación básica y superior pública de todos los sistemas de enseñanza y, para el plan de Carrera de los(as) profesionales de la educación básica pública, tomar como referencia el Salario Mínimo nacional profesional, definido en ley federal (BRZEZINSKI, 2011, p. 5).

Seleccionamos para análisis de la valoración de los profesionales de la educación las estrategias 18.2 y 18.4 por postular la formación y ejercicio profesional, con vínculos intrínsecos a las políticas de formación en nivel superior (graduación y postgrado) y a la educación básica.

Estrategia 18.2) implantar, en las redes públicas de educación básica y superior, acompañamiento de los profesionales iniciantes, supervisados por equipo de profesionales con experiencia, a fin de fundamentar, con base en evaluación documentada, la decisión por la efectuación tras el periodo probatorio y ofrecer, durante ese período, curso de perfeccionamiento de estudios en el área de actuación del (de la) profesor(a), con foco para los contenidos a ser enseñados y las metodologías de enseñanza de cada disciplina (BRZEZINSKI, 2011, p. 5).

El acompañamiento de profesores iniciantes por equipos de profesionales con experiencia es una de las modalidades de formación continuada que contribuye significativamente para la profesionalización docente y que deberá hacerse realidad en el sistema educacional brasileño como proclamado en esa estrategia.

Cuestionamos, sin embargo, por qué no realizarlo inmediatamente tras el ingreso del profesor en el sistema de enseñanza vía concurso público de pruebas y títulos. En la trayectoria del periodo probatorio, el recién ingresado se siente sin preparación para enfrentar la complejidad del acto docente y el conocimiento in loco de la organización de la educación escolar, una vez que son muchas dificultades para ejercer la praxis pedagógica. Esos obstáculos, pueden suscitar sentimientos de incapacidad cuando el profesor iniciante se pone en conflicto con la inmersión en la realidad de la sala de aula y en el contexto escolar, un cotidiano pleno de diversidad y heterogeneidades. Muchos no ultrapasan esos conflictos. Por analogía las ideas de Fernando Pessoa (2014) afirmamos que el profesor iniciante tiene un arduo recorrido, "es el tiempo de la travesía: y, si no osamos a hacerla, nos habríamos quedado, para siempre, al margen de nosotros mismos".

En la estrategia 18.4, en el PNE el legislador retoma un importante aspecto de la profesionalización docente prescrito en la LDB/1996 (art. 67), entretanto, sin aplicación sistemática por los sistemas de enseñanza. El aparato legal se refiere a la prescripción en los planes de carrera de los profesionales de la educación de licencia remunerada e incentivos en la modalidad de becas de estudios o de otra forma de apoyo institucional para cualificación tanto en el lato como en el stricto sensu. Ese derecho del profesor en ejercicio en los sistemas públicos de educación básica está garantizado también en la Meta 16 (PNE 2014-2024).

Discutida la formación y valoración de los profesionales de la educación en el PNE del Brasil, el foco se orienta hacia al análisis del Plan Andaluz de Formación del Profesorado, objetivo del ítem 4, a seguir.

\section{ITINERARIO INVESTIGATIVO: III PLAN ANDALUZ DE FORMACIÓN DEL PROFESORADO (2014)}

El Plan Andaluz de Formación Permanente del Profesorado que analizamos es el tercero que se publica en Andalucía. El primer plan se publicó en 1992 y el segundo en 2003. Por lo tanto, aunque no se indica expresamente, cada plan tiene una duración aproximada de 10 años. El III Plan considera de nuevo a la formación 


\section{autêntica}

permanente del profesorado como un factor clave para la mejora de la calidad de la educación en Andalucía. Más concretamente afirma que

la formación del profesorado constituye un elemento fundamental para dar respuesta a los nuevos retos educativos que plantea la sociedad actual, puesto que se trata del factor clave para conseguir la mejora de la competencia profesional de los docentes, lo que contribuirá, en consecuencia, al desarrollo de una enseñanza de calidad y equidad (ESPAÑA.ANDALUCíA, 2014).

La formación permanente se plantea centrada en la escuela, entendiendo que es la institución educativa el espacio de donde deben surgir las necesidades de formación, innovación y mejora.

a) promover la mejora de la institución escolar como organización educativa;

b) transformar la cultura organizativa compartimentada de los centros en cultura de colegialidad y trabajo compartido;

c) repensar el por qué, para qué y cómo de la educación por parte de un equipo de profesionales que trabajan juntos en un centro y atienden al mismo alumnado;

d) facilitar un espacio común para la reflexión;

y) convertir el centro en elemento básico de mejora, formación e innovación, totalmente contextualizadas y con la posibilidad de su aplicación inmediata en las aulas (ESPAÑA. ANDALUCíA, 2014).

Para conseguir estos objetivos, el III Plan de Formación Permanente del profesorado plantea las siguientes líneas estratégicas de formación:

I. La formación del profesorado vinculada a la mejora de las prácticas educativas, el rendimiento y el éxito educativo de todo el alumnado.

II. La formación del profesorado como factor clave para el perfeccionamiento continuo y la capacitación profesional docente.

III. La formación del profesorado como impulsora del conocimiento compartido y producido en los centros educativos, de la investigación y la innovación educativa y de las buenas prácticas

IV. La formación del profesorado como apoyo a la progresiva transformación de los centros educativos en entornos colaborativos de aprendizaje y formación en los que participan todos los miembros de la comunidad educativa.

V. La formación del profesorado de Formación Profesional, enseñanzas Artísticas, enseñanzas Oficiales de Idiomas y educación Permanente como herramienta para conectar la educación con la realidad productiva y el empleo (ESPAÑA. ANDALUCÍA, 2014).

Vamos a ir describiendo cada una de estas líneas estratégicas.

\subsection{LÍNEA I. LA FORMACIÓN DEL PROFESORADO VINCULADA A LA MEJORA DE LAS PRÁCTICAS EDUCATIVAS, EL RENDIMIENTO Y EL ÉXITO EDUCATIVO DE TODO EL ALUMNADO}

El III Plan entiende que la formación del profesorado "está vinculada a la práctica educativa, de tal manera que incida directamente en ella, con una repercusión concreta y positiva en la formación y el rendimiento del alumnado, y es con esta premisa como deben contemplarse las demandas formativas". Así, la formación debe tomar como eje central mejorar la capacidad de enseñar y reflexionar de los docentes. Para ello toma en consideración las necesidades de aprendizaje de los estudiantes. 


\section{autêntica}

Para conseguir estos objetivos, el plan propone el desarrollo de los siguientes ejes de formación:

1. La organización del currículo basado en las competencias clave.

2. Escuela inclusiva para la igualdad y la equidad: atención a la diversidad, convivencia e igualdad.

3. Escuela de la sociedad del conocimiento, destrezas básicas: plurilingüismo, uso de las TIC y espíritu emprendedor.

4. Planes de mejora y de formación de los centros educativos.

\subsection{LÍNEA II. LA FORMACIÓN DEL PROFESORADO COMO FACTOR CLAVE PARA EL PERFECCIONAMIENTO CONTINUO Y LA CAPACITACIÓN PROFESIONAL DOCENTE}

Esta línea de actuación se centra principalmente en el docente y su desarrollo profesional. En España no existe un sistema de evaluación ni de carrera docente. Por ello se espera que la formación promueva el desarrollo de competencias que los docentes requieren. Se afirma que:

\footnotetext{
La formación del profesorado, desde que se inicia en la práctica educativa hasta el final de su vida laboral, debe perseguir el desarrollo de las competencias profesionales de los docentes vinculado a la mejora de la calidad de la enseñanza, dado que no todos los puestos de trabajo docentes requieren de las mismas competencias, se distingue la formación para las competencias comunes, que debe de tener todo docente, al ser las mismas para todo puesto de trabajo, y formación para las competencias especificas, vinculadas a los diferentes perfiles profesionales (docencia, orientación, formación, inspección, dirección y coordinación) (ESPAÑA.ANDALUCÍA, 2014)
}

Los ejes de formación que conforman esta línea son de los principalmente:

1. Desarrollo profesional adecuado al rol profesional: competencias comunes y competencias específicas.

2. Desarrollo profesional a lo largo de toda la vida profesional: formación inicial, profesorado novel, formación permanente.

\subsection{LÍNEA III. LA FORMACIÓN DEL PROFESORADO COMO IMPULSORA DEL CONOCIMIENTO COMPARTIDO Y PRODUCIDO EN LOS CENTROS EDUCATIVOS, LA INVESTIGACIÓN Y LA INNOVACIÓN EDUCATIVA Y LAS BUENAS PRÁCTICAS}

La línea III pone énfasis en la necesidad de desarrollar en los centros educativos innovaciones e investigaciones que promuevan la producción de conocimiento basado en la práctica. Para ello se plantea la necesidad de promover la concepción del aula y el centro como espacios para la innovación, investigación y la formación y de impulsar la creación de redes de centros como elemento dinamizador de la innovación del profesorado.

Por otra parte, conseguir escuelas innovadoras requiere que el profesorado esté capacitado en los procesos de innovación y de investigación. Para ello, el III Plan pretende promover la capacitación del profesorado la través de actuaciones educativas investigadoras, analíticas y críticas de las prácticas educativas y propiciar la interacción entre el profesorado la través de metodologías de trabajo colaborativas.

En esta línea prioritaria de formación se destacan de los grandes ejes de formación:

1. Investigación e innovación educativa. Desarrollo de programas educativos. 


\title{
autêntica
}

2. Metodologías de trabajo investigador y colaborativo en el aula y centro.

\begin{abstract}
4.4 LÍNEA IV. LA FORMACIÓN DEL PROFESORADO COMO APOYO A LA PROGRESIVA TRANSFORMACIÓN DE LOS CENTROS EDUCATIVOS EN ENTORNOS COLABORATIVOS DE APRENDIZAJE Y FORMACIÓN EN LOS QUE PARTICIPAN TODOS LOS MIEMBROS DE LA COMUNIDAD EDUCATIVA
\end{abstract}

El III Plan Andaluz de Formación Permanente del Profesorado establece que la formación y el desarrollo de programas y planes educativos deben obedecer a proyectos colectivos que integren al mayor número posible de miembros de la comunidad educativa. El conocimiento compartido se instaura en el centro, mientras que el individual se va con la persona que lo ha generado.

\begin{abstract}
La cultura organizativa de un centro educativo debe entenderse hoy desde una perspectiva conjunta de trabajo colaborativo y en equipo. Parte de esa cultura consiste en hablar, escribir y describir la vida del centro para que se haga evidente a todos sus miembros, para después repensar las prácticas e implementar cambios coherentes. La comunidad educativa debe integrar las voces de todos sus miembros en el proyecto educativo de centro (ESPAÑA.ANDALUCíA, 2014).
\end{abstract}

Esta propuesta se concreta en los siguientes objetivos:

- Integrar a todos los miembros de la comunidad educativa en las actuaciones formativas dirigidas o fomentar su participación en el centro.

- Dar a conocer los modelos de participación, intervención y desarrollo comunitario

- Establecer y utilizar canales de comunicación y participación entre el centro educativo, la comunidad educativa y el entorno.

Para conseguir estas metas se ponen en marcha los siguientes ejes de formación:

1. La participación de toda la comunidad para la mejora de los rendimientos escolares y la gestión del centro.

2. La atención a los centros con entornos de aprendizaje específicos (compensación, rural, hospitalarios, centros específicos de educación especial, etc.).

\section{5 LÍNEA V. LA FORMACIÓN DEL PROFESORADO DE FORMACIÓN PROFESIONAL, ENSEÑANZAS ARTÍSTICAS, ENSEÑANZAS OFICIALES DE IDIOMAS Y EDUCACIÓN PERMANENTE COMO HERRAMIENTA PARA CONECTAR LA EDUCACIÓN CON LA REALIDAD PRODUCTIVA Y EL EMPLEO}

Esta última línea de actuación tiene relación con los compromisos y responsabilidades de España como miembro de la Unión Europea. Los objetivos de la Unión Europea en la estrategia educativa 2020, establecen la necesidad de una mayor coordinación entre el mundo de la escuela y el mundo del trabajo. Esto se hace especialmente importante en los profesionales de la educación que ejercen su labor docente con alumnado de ciclos de Formación Profesional, enseñanzas Artísticas, de Idiomas y educación Permanente.

El profesorado que imparte estas especialidades ha de contar también con el apoyo del sistema andaluz de formación, por lo que se requiere una atención especial, dadas sus características de especialización.

El III Plan Andaluz de Formación Permanente del Profesorado contempla la necesidad de una evaluación continua del plan. Así, se establece que 


\section{autêntica}

la evaluación se efectuará sobre los procesos formativos y sobre los resultados obtenidos, tanto en lo relativo a la organización y gestión de las actividades formativas, como su impacto en la práctica docente del profesorado y en la mejora de los resultados académicos del la alumnado (ESPAÑA. ANDALUCÍA, 2014).

La evaluación se realiza tanto desde el punto de vista cuantitativo como cualitativo y busca generar indicadores para conocer en qué medida la formación incide en las prácticas educativas y su repercusión en los resultados de aprendizaje del alumnado.

\section{APROXIMACIONES Y DISTANCIAMIENTOS ENTRE EL PNE/BR (2014-2024) Y EL PLAN ANDALUZ DE FORMACIÓN DEL PROFESORADO}

Llegamos al momento de presentar los resultados de la investigación en este artículo. Perduró, no obstante, una indagación entre nosotros, autores: ¿Es posible hacer comparaciones entre un país con 205 millones de habitantes (IBGE, 2016) y una autonomía como Andalucía con 8 millones de habitantes? Nos atrevemos a establecer algunas comparaciones a riesgo de ser criticados por las enormes diferencias existentes entre nuestros países.

En la tentativa de superar nuestra inquietud recorremos a la asertiva arriba citada de Garrido García (1991, p.157), estudiado por Trojan y Sanchez (30 mar. 2009): "para la educación comparada, aunque se contemplen diferentes enfoques, su objeto comprehende los sistemas de educación, o la consideración del proceso de educación en sistemas sociales concretos."Tras ello nos tranquilizamos, pues, aunque sean cuantitativamente distantes en términos de habitantes, Brasil y Andalucía, nuestro objeto de comparación son las políticas de formación y valoración de profesores y revelamos las políticas concretas establecidas en planes que tienen duración decenal. He aquí una aproximación independientemente de la cantidad de habitantes: los planes educacionales del Brasil y de Andalucía tienen una duración de diez años, aproximadamente.

Otra aproximación recae en el aspecto que caracteriza a Andalucía es que, desde 1982, está gobernada de forma ininterrumpida por el Partido Socialista Obrero Español. Esta permanencia en el poder - mantenido de forma democrática - durante un largo período de tiempo (34 años) ha permitido consolidar políticas educativas propias de un partido socialdemócrata. En Andalucía, en torno al $76 \%$ de los centros educativos son públicos, el $20 \%$ concertados (privados con financiación pública) y sólo el $4 \%$ son privados. Además, la formación continua en Andalucía tiene políticas permanentes y gratuitas.

Estos números se han ido manteniendo con bastante consistencia a lo largo de los años, lo que ha permitido que la disputa entre lo público y lo privado en lo que respecta a la formación permanente del profesorado en Andalucía haya sido un tema relativamente ausente. Sí es cierto que, debido a la crisis económica presente en España desde 2008, los recursos financieros destinados a la educación han bajado y han forzado que muchos profesores dejen el sistema.

Brasil es gobernado por un régimen político socialdemócrata, como España. El distanciamiento entre los sistemas de gobierno reside exactamente en la diferencia entre un régimen político Republicano Presidencialista Democrático y Representativo (BR) y una Monarquía Constitucional y Representativa.

Otro distanciamiento evidenciado es que en Andalucía se constata que la disputa de recursos financieros destinados a la enseñanza pública y privada es un aspecto relativamente ausente, al paso que en Brasil 


\section{autêntica}

se mantiene una lucha enconada por los sujetos sociales colectivos a favor de destinar recursos públicos exclusivamente para instituciones públicas y gratuitas. El gobierno popular brasileño continúa destinando recursos para la enseñanza privada en detrimento de la enseñanza pública, como revela la evaluación de la Meta 20 y su Estrategia 20.12 del PNE/2014-2024.

Es interesante que en España no exista un sistema nacional de evaluación ni de carrera docente, mientras que en el Brasil hay un consolidado sistema de evaluación de cursos, de instituciones, de profesores, del alunado, con exámenes estandarizados, cuyo "ranking" se hace con base en datos divulgados por el Instituto de Estudios e Investigaciones Educacionales Anísio Teixeira y por la Coordinación de Perfeccionamiento de Personal de Nivel Superior (Capes). En esa particularidad se registra un distanciamiento.

En lo que respecta al sistema de acceso del profesorado (tanto de escuela pública como privada), todo el profesorado ha de estar en posesión de un título profesional universitario para poder ejercer la función docente en Andalucía. No existen docentes enseñando en escuelas sin la formación exigida. En Brasil se asegura por la legislación que la formación inicial deberá ser hecha en cursos de licenciatura, por lo tanto en nivel superior, y que el ingreso en la enseñanza pública debe ser por oposiciones. También debe ser considerada la compatibilidad de actuación en el área para la cual los profesores fueron formados. Contradictoriamente, ninguno de esos dispositivos legales es respetado.

Hay profesores en Brasil en las escuelas públicas y privadas que todavía no tienen diploma de Magisterio en nivel de Enseñanza Media y los concursos públicos no son realizados con regularidad para la necesidad demandada, lo que impide que el profesional pueda mantener un vínculo laboral en el sistema educacional brasileño de forma efectiva.

Aunque los distanciamientos sean muchos, tenemos claro que una gran aproximación fue constatada en lo que atañe a las orientaciones de la formación permanente del profesorado. En este sentido, la situación en Andalucía puede equipararse a la descrita por CONAE/2010, acerca de los principios de la Base Común Nacional construida por la Anfope, para la formación de todos los que aspiran a ser profesores: la) sólida formación teórica e interdisciplinar; b) unidad teoría-práctica; c) trabajo colectivo e interdisciplinar; d) compromiso social del profesional de la educación; e) gestión democrática; f) incorporación de la concepción de formación continuada; g) evaluación permanente de los cursos de formación de los profesionales de la educación. Estos principios son coincidentes con los expuestos en el III Plan Andaluz de Formación Permanente del Profesorado. 


\section{autêntica}

\section{CONSIDERACIONES CONCLUSIVAS}

Las políticas de formación permanente del profesorado indican qué prioridades adoptan los responsables de diseñar y desarrollar las políticas educativas (Vaillant y Marcelo, 2015). Estas políticas determinan, facilitan o impiden el desarrollo de una formación profesionalizada o bien burocrática. La formación debe entenderse como un derecho pero también como un deber de los profesores. Un derecho que debe preservarse a través de una oferta de formación atractiva, cercana a las necesidades de los docentes, de las escuelas y de los alumnos. Una formación que tome en consideración el entorno de trabajo del docente y facilite acciones de formación comprometidas con la mejora de la práctica (Marcelo y Vaillant, 2009).

En este proceso un aspecto importante es la existencia o no de un sistema de formación permanente que dé cobertura y estructura de funcionamiento. Cuando no existe un sistema de formación, ésta queda relegada a las iniciativas de instituciones normalmente externas a las escuelas. Principalmente las universidades. La formación permanente del profesorado debería ser una preocupación de las universidades, pero lo que percibimos es que a menudo las universidades ofertan aquello que creen necesitan los docentes pero no lo que reamente demandan. Cuando la formación se mide sólo en número de horas de formación a través de cursos, diplomados o magísteres los aspectos importantes quedan fuera.

Pero la formación requiere también del compromiso del propio profesorado. Decíamos que la formación es un derecho pero también un deber. Un deber que no puede ser obligado porque las ciencias del aprendizaje nos enseñan que las personas no aprenden si no tienen motivación para aprender. El deber de formarse es una seña constitutiva de la identidad de los profesionales. Y los docentes deben de asumir que la formación inicial no es suficiente para desempeñar las complejas funciones de la escuela del siglo XXI. Una escuela que demanda profesionales con capacidad de autonomía en el aprendizaje, de autoformación.

La formación, así es un proceso que empodera a los docentes para poder dar respuesta a las necesidades de las escuelas y algunos. Y a menudo la formación no es algo que se da, sino que se construye individualmente y en equipo. La formación del profesorado es un campo de ideología y lucha, pero también de proyectos, programas, actuaciones que se diseñan, desarrollan y evalúan. 


\section{autêntica}

\section{REFERÊNCIAS}

AMARAL, N, C. Fontes para o financiamento da educação pública brasileira na visão dos $10 \%$ do PIB. Anais do XII Encontro de Pesquisa em Educação Centro Oeste. Goiânia: PUC Goiás, 19 a 22 de out. 2014.

ANTUNES, Ricardo. Adeus ao Trabalho: ensaios sobre as metamorfoses e a centralidade do mundo do trabalho. 12 ed. São Paulo: Cortez, 2007.

Os sentidos do Trabalho: ensaio sobre a afirmação e a negação do trabalho. 2 ed. São Paulo: Boitempo, 2009.

BRASIL/PR/MEC. Lei n. 13.005, de 25/6/2014 (PNE 2014-2024). Aprova o Plano Nacional de Educação. Disponível em: <http://www.planalto.gov.br/ccivil_03/_Ato2011-2014/2014/Lei/L13005.htm>. Acesso em: 15 out. 2014.

BRASIL/MEC/SEA. Documento final CONAE/2010. Disponível em: <http://conae.mec.gov.br/images/stories/ pdf/pdf/documetos/documento_final_sl.pdf>. Acesso em: 29 jul.2015.

BRZEZINSKI, I. Política de formação de professores: a formação do professor dos anos iniciais do Ensino Fundamental, desdobramentos em dez anos da Lei 9.934/1996. In. BRZEZINSKI, I. (Org.) LDB dez anos depois. Reinterpretação sob diversos olhares. 9 ed. São Paulo: Cortez Editora, 2012, p. 195-219

Fundamentos e contribuições à elaboração do plano nacional de educação (2011-2022) com base nas emendas ao PI 8.035/2011. Pronunciamento. Brasília: Anais da Comissão de Educação da Câmara dos Deputados, 31/8/2011.

CANÁRIO, R.; RUMMERT, S. M.. Educação de jovens e adultos trabalhadores no Brasil e em Portugal: escritos preliminares. In: SOUZA, D. B. de; MARTÍNEZ, S. A.. Educação comparada: rotas de além-mar. São Paulo, Xamã, p. 355-371.

CASTELLS, M.. O poder da identidade.Trad. Klauss B. Gerhardt. São Paulo: Paz e Terra, 1999.

CAVALCANTE, M. P.. A cidadania como campo de disputa das classes subalternas. Anais da I/ Jornada Internacional de Políticas Públicas. São Luis: UFM, 2005, p.1-8.

CHAUÍ. M.. Convite à filosofia. 13 ed. São Paulo: Ática, 2008.

COUTINHO, C. N.. 2 ed. rev. Contra a corrente: ensaios sobre democracia e socialismo. São Paulo: Cortez, 2008.

Notas sobre cidadania e modernidade. Revista de Estudos de Política e Teoria Social. Rio de Janeiro:

UFRJ/DP\&A, v. 1, n. 1, p. 145-165, 1997.

DIAS, E. F. A liberdade (im) possível na ordem do capital: reestruturação produtiva e passivização. São Paulo:

IFCH/UNICAMP, 1997. 


\section{autêntica}

Cidadania e racionalidade de classe. Universidade e Sociedade, São Paulo: ANDES-SN, n. 11, p.130139, 1996.

ESPANHA. Andalucía. III Plan Andaluz de Formación del Profesorado. Orden de 31 de julio de 2014. Disponível em: http://www.juntadeandalucia.es/boja/2014/170/2. Acesso em: 12 jan. 2016.

Ley 17 de 10 de diciembre de 2007, de Educación de Andalucía. Disponível: http://noticias.juridicas. com/base_datos/CCAA/an-l17-2007.html. Acesso em: 1 jan. 2016.

FONTES, V. O Brasil e o capital-imperialismo. Teoria e história. Rio de Janeiro: FIOCRUZ/UFRJ Editora, 2010.

GARCIA GARRIDO, J. L.. Fundamentos de Educación Comparada. 3 ed. Madri: Dykinson, 1996.

GOHN, M. da G.. Teorias dos movimentos sociais: paradigmas clássicos e contemporâneos. São Paulo: Loyola, 1997.

IANNI, O., Teorias da globalização.3 ed. Rio de Janeiro: Civilização Brasileira, 1996.

MARCELO, C.; VAILLANT, D. Desarrollo professional docente. Madrid: Narcea, 2009.

MARCELO, C. Desenvolvimento Profissional Docente: passado e futuro. Sísifo. n. ${ }^{\circ}$ 8, p. 7-22, 2009.

MENEZES JUNIOR; A. da S.; BRZEZINSKI, I.. Políticas curriculares na formação médica: aproximações e distanciamentos entre Brasil e Portugal. Trabalho, Educação e Saúde. Revista Fiocruz, 2015.

MINISTERIO DA FAZENDA.TESOURO NACIONAL. Classificação de risco da República Soberana do Brasil. Disponível em: http://www.tesouro.fazenda.gov.br/classificacao-de-risco.Acesso em: 2 jan. 2016

NÓVOA, A.. Modelos de análise de educação comparada: o campo e o mapa. In: SOUZA, D. B. de; MARTíNEZ, S. A.. Educação comparada: rotas de além-mar. São Paulo, Xamã, 2009, p.23-62.

OLIVEIRA, D. A.. Os docentes no Plano Nacional de Educação: entre a valorização e a desprofissionalização docente. Retratos da Escola, v.8, n.15, jul./dez.2014, p. 447-460

PESSOA, F. É o tempo da travessia. Disponível em: <http://kdfrases.com>. Acesso em: 20 set 2014.

REVISTA EXAME (on line). Brasil é a 14 a economia do mundo, segundo Banco Mundial. Disponível em: < http://exame.abril.com.br/economia/noticias/brasil-e-a-14a-economia-do-mundo-segundo-banco-mundial-m0083015>. Acesso em: 15 out 2015.

RUMMERT, S. M.; ALGEBAILE, E.; VENTURA, J.. Educação da classe trabalhadora brasileira: expressão do desenvolvimento desigual e combinado. Rev. Bras. Educ. [online]. 2013, vol.18, n.54, pp. 717-738. ISSN 1413-2478. http://dx.doi.org/10.1590/S1413-24782013000300011.

SADLER, S. M.. How far can we learn anything of practical value from the study of foreign systems of educacion? Comparative Education Review, v.7, n.3, p.307-314, 1900. 


\section{autêntica}

TROJAN, R. M.; SANCHEZ, M. M.. Educação comparada: considerações teórico-metodológicas no contexto da globalização. Disponível em: htpp://www.saece.org.ar/docs/congreso3/Trojan2.doc. Acesso em 5 mai. 2015.

YANG, R.. Comparações entre políticas. In: BRAY, M.; ADMSON, B.; MASSON, M. Pesquisa em educação comparada: abordagens e métodos. Brasília: Liberlivro, 2015.

ZEMELMAN, H.. Algunas reflexiones metodológicas a partir del problema de las investigaciones comparativas. In: KRAWCZYK, N. R.; WANDERLEY, Luiz E.. (Orgs). América Latina: Estado e reformas numa perspectiva comparada, São Paulo: Cortez, 2003.

VAILLANT, D.; MARCELO, C. El A, B, C y D de la formación docente. Madrid: Narcea, 2015.

WISEMAN, A. W. The uses of evidence for educational policymaking; global contexts and international trends. Review of Researd in Education. v. 34, n.1, p. 1-24, 2010 